\title{
STRIPPER FOIL TEMPERATURES AND ELECTRON EMISSION AT THE LOS ALAMOS PROTON STORAGE RING*
}

\author{
T. Spickermann ${ }^{\dagger}$, M. Borden, A. Browman, D. Fitzgerald, R. Macek, R. McCrady, T. Zaugg, \\ Los Alamos Neutron Science Center, LANL, Los Alamos, NM 87545, USA
}

\section{Abstract}

We have modeled the heating process of the PSR stripper foil and compared our results to observations that depend on the foil temperature. The foil is heated by the energy deposited by injected $\mathrm{H}^{-}$ions and stored protons passing through the foil. Secondary emission of electrons due to these foil hits results in a measurable current that we can use to benchmark our model. At higher beam intensities thermionic emission of electrons dominates the foil current. Due to the extreme temperature dependence of the thermionic current this is a very sensitive indicator of the foil temperature and will be used to safeguard against overheating the foil in extreme beam conditions. We will present our best estimates of the foil temperature for different beam intensities.

\section{SIMULATION OF FOIL HEATING}

To inject protons into the Proton Storage Ring (PSR) $\mathrm{H}^{-}$ ions with a kinetic energy of $800 \mathrm{MeV}$ pass through a carbon foil [1], typically of an area density of $400 \mu \mathrm{g} / \mathrm{cm}^{2}$, where the two electrons are stripped off. Injection pulse lengths are typically of the order of a millisecond, or several thousand turns of $359 \mathrm{~ns}$ duration. Injection is "off-axis", i.e. off the closed orbit in the ring ("On-axis" injection is occasionally used during beam studies). A four-magnet vertical closed orbit bump is used to "paint" the injected beam over the available phase space in the ring to reduce space charge effects and to reduce the number of foil hits by the circulating beam. The stripper foil position is adjusted to cover about $97-98 \%$ of the injected beam. Totally covering the injected beam would approximately double the number of foil hits by the circulating beam and consequently double the beam loss rate. Observing the stripper foil with a video camera shows bright flashes during the accumulation cycle, indicating that the foil gets "red-hot". Presently installed equipment does not allow, however, measurement of the foil temperature with any precision. Instead, we use the ORBIT [2] code to simulate the accumulation and storage of protons in the PSR. This provides us with the number of foil hits per time. Each circulating proton hits the foil about 30-80 times (depending on beam conditions) during the accumulation cycle. The average energy deposited per foil hit can be obtained from stopping power tables [3].

\footnotetext{
* Work conducted at the Los Alamos Laboratory, which is operated by the University of California for the United States Department of Energy under contract W-7405-ENG-36.

† Email: spickermann@1anl.gov
}

\section{FOIL CURRENT}

While we lack a direct measurement of foil temperature we do measure the foil current. Figure 1 shows the measured current for a final beam charge of $8.7 \mu \mathrm{C}$ injected over $1225 \mu$ s.

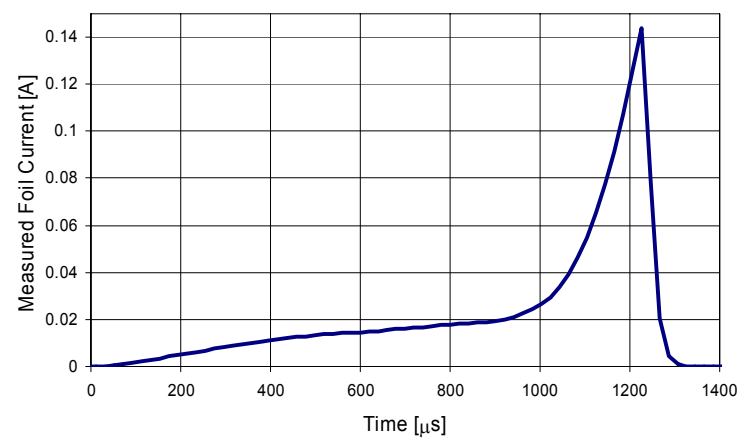

Figure 1: Measured foil current. Beam is extracted immediately after injection at $1225 \mu \mathrm{s}$.

From start of injection to $\sim 900 \mu$ s the current is dominated by secondary emission. It is therefore directly proportional to the total number of foil hits per time and can be used as a check on the ORBIT simulation. The secondary emission yield $\mathrm{Y}$, i.e. the average number of electrons emitted per proton hitting the foil can be estimated with the Sternglass formula [4]:

$\mathrm{Y}=\frac{\mathrm{Pd}_{\mathrm{S}}}{\mathrm{E}_{*}} \cdot \frac{\mathrm{dE}}{\mathrm{dx}} \quad$ (per surface),

where $\mathrm{P}$ is a probability $(\sim 0.5), \mathrm{d}_{\mathrm{s}}$ is the average depth from which secondaries arise $(\sim 1 \mathrm{~nm})$ and $E_{*}$ is the average amount of kinetic energy lost by a proton per ionization $(\sim 25 \mathrm{eV})$. Here, $\mathrm{dE} / \mathrm{dx}$ is in $\mathrm{eV} / \mathrm{nm}$. At $\mathrm{t}>$ $900 \mu$ s thermionic emission (TE) becomes the dominant contributor to the foil current.

\section{ENERGY EQUATION}

To calculate the maximum temperature we have divided the foil area in the model into bins of $1.5 \cdot 1.5 \mathrm{~mm}^{2}$. The peak temperature in the "hottest bin", i.e. the bin with the maximum number of hits can be calculated by solving the energy equation 


$$
\begin{aligned}
\Delta \mathrm{E} \frac{\mathrm{dN}_{\text {hits }}}{\mathrm{dt}} & =\rho \mathrm{Vc}(\mathrm{t}) \frac{\mathrm{dT}}{\mathrm{dt}}-2 \mathrm{~A} \varepsilon \sigma\left(\mathrm{T}^{4}-\mathrm{T}_{\text {amb }}^{4}\right) \\
& -2 \mathrm{AJ}_{\mathrm{TE}}\left(\mathrm{A}_{\mathrm{R}}, \varphi, \mathrm{T}\right) \cdot\left(\varphi+\frac{2 \mathrm{kT}}{\mathrm{q}}\right)
\end{aligned}
$$

Table 1 shows the variables used in Eq. 1.

Table 1:

\begin{tabular}{|l|l|l|}
\hline Var. & Value & Description \\
\hline$\rho$ & $2.0 \mathrm{~g} / \mathrm{cm}^{3}$ & Density of carbon \\
\hline $\mathrm{c}$ & function of temperature & Heat capacity of carbon \\
\hline$\sigma$ & $5.67 \cdot 10^{-8} \mathrm{~J} /\left(\mathrm{s} \mathrm{m}^{2} \mathrm{~K}^{4}\right)$ & Stefan-Boltzmann constant \\
\hline$\varepsilon$ & 0.8 & Emissivity \\
\hline $\mathrm{q}$ & $1.602 \cdot 10^{-19} \mathrm{C}$ & Electron charge \\
\hline $\mathrm{A}_{\mathrm{R}}$ & $\begin{array}{l}120 \mathrm{~A} / \mathrm{K}^{2} \mathrm{~cm}^{2} \\
\text { (theoretical value })\end{array}$ & Richardson constant \\
\hline$\varphi$ & $\sim 4.5 \mathrm{eV}$ & Work function \\
\hline $\mathrm{k}$ & $1.38 \cdot 10^{-23} \mathrm{~J} / \mathrm{K}$ & Boltzmann constant \\
\hline$\Delta \mathrm{E}$ & $825 \mathrm{eV}$ & Energy deposited per hit \\
\hline $\mathrm{N}_{\text {hits }}$ & function of time & Number of hits in bin \\
\hline $\mathrm{A}$ & $2.25 \mathrm{~mm}^{2}$ & Area of one bin \\
\hline $\mathrm{V}$ & $4.5 \cdot 10^{-3} \mathrm{~mm}^{3}$ & Volume of one bin \\
\hline $\mathrm{T}_{\mathrm{amb}}$ & $297 \mathrm{~K}$ & Ambient temperature \\
\hline $\mathrm{J}_{\mathrm{TE}}$ & Function of temperature & Thermionic current density \\
\hline
\end{tabular}

Cooling via heat conduction was not included in the calculation. However, solving the heat equation for a simplified geometry shows that conduction would lower the peak temperature by less than $1 \%$.

\section{Foil Heating}

From the ORBIT simulation we obtain the number of hits per turn to which we fit a piecewise linear function to obtain the number of hits as a function of time. The simulation also tells us where on the foil each hit occurs. This allows us to divide the foil into bins and to calculate the number of foil hits in each bin. Multiplied with the average energy deposit per foil hit $\Delta \mathrm{E}$ this gives the energy deposited per time for each bin.

\section{Heat Capacity}

The heat capacity of carbon is a strong function of temperature $[5,6]$. This must be taken into account when solving the energy equation. The sharp increase of the heat capacity with temperatures above room temperature $(297 \mathrm{~K})$ results in a slower rise in the foil temperature, but also in a slower cooling process after beam extraction.

\section{Thermionic Emission}

Thermionic Emission is expected to play a role at extreme foil temperatures, due to the very strong temperature dependence of thermionic currents. The thermionic current density can be calculated as

$$
J\left(A_{R}, \varphi, T\right)=A_{R} T^{2} e^{\frac{-q \varphi}{k T}} .
$$

The power dissipated by thermionic emission is given by [7]

$$
\frac{\mathrm{dE}_{\mathrm{TE}}}{\mathrm{dT}}=2 \mathrm{~A} \cdot\left(\varphi+\frac{2 \mathrm{kT}}{\mathrm{q}}\right) \cdot \mathrm{J}_{\mathrm{TE}}\left(\mathrm{A}_{\mathrm{R}}, \varphi, \mathrm{T}\right) .
$$

\section{PEAK FOIL TEMPERATURES}

Solving the energy equation Eq. 1 is an iterative process because some parameters are not well known, e.g the number of foil hits, which depends on the exact foil position with respect to the injected beam, or the parameters that go into the calculation of the thermionic current. These have to be adjusted to obtain the best possible agreement between the measured foil current and the current calculated as the sum of secondary emission and thermionic emission currents. Figure 2 shows a comparison between a measured current and the current obtained from the model for a beam intensity of $8.7 \mu \mathrm{C} /$ pulse injected over $1225 \mu$ s with a pulse repetition rate of $4 \mathrm{~Hz}$.

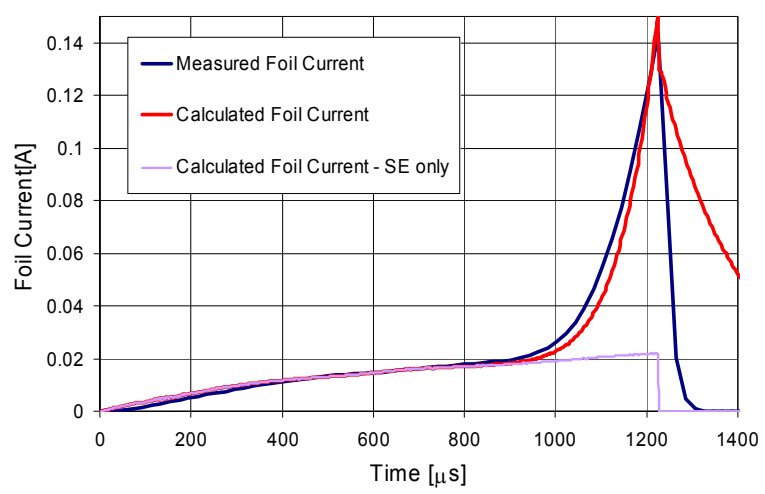

Figure 2: Comparison of measured and calculated foil currents.

The TE current was calculated with $\varphi=4.5 \mathrm{eV}$ and $\mathrm{A}_{\mathrm{R}}=2.2 \mathrm{~A} /\left(\mathrm{cm}^{2} \mathrm{~K}^{2}\right)$. Better agreement between the measured and calculated currents could be achieved with lower values for the work function $\varphi$, but these are not supported by literature. One also notices that after beam extraction the measured current drops much faster than the calculated one. We believe that this may be due to space charge built up around the foil by TE electrons as these are no longer removed by the strong potential of the circulating beam. We plan to test this hypothesis in the coming run period by biasing the foil. One should note that, unless the measured current is from the very first injected beam pulse, one has to solve the energy equation for consecutive pulses and cool down times (given by the pulse repetition rate) because the initial foil temperature on subsequent pulses will be higher than the ambient temperature. For this case we calculate a maximum temperature of $3122 \mathrm{~K}$ in the hottest bin in the foil. Neglecting thermionic emission yields a value only $\sim 1 \mathrm{~K}$ 
higher. Although it may dominate the foil current, TE is not a significant source of cooling for the foil.

\section{Peak Temperatures for Production Beams}

With the parameters of the simulation and calculation adjusted one can compute the foil temperature for different beam conditions. Figure 3 shows the temperature in the hottest bin for a production beam, i.e. $125 \mu \mathrm{A}$ with a pulse repetition rate of $20 \mathrm{~Hz}$ and for beam conditions expected in a potential upgrade, i.e. $200 \mu \mathrm{A}$ at $30 \mathrm{~Hz}$. Although the beam intensities are not too different $(6.25 \mu \mathrm{C} /$ pulse vs. $6.7 \mu \mathrm{C} /$ pulse) the higher repetition rate leads to a significantly higher peak temperature.

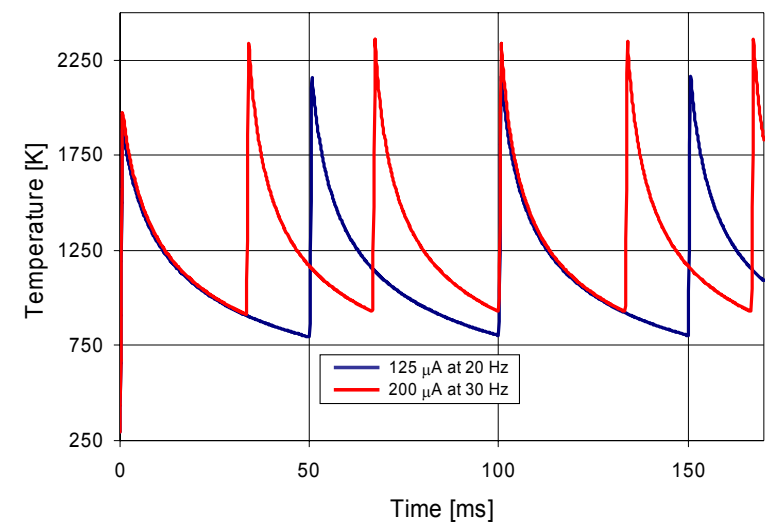

Figure 3: Temperatures in hottest bin for a productiontype beam and for a planned upgrade.

The peak temperatures are $2182 \mathrm{~K}$ for the productiontype beam and $2361 \mathrm{~K}$ for the upgrade. Both peak temperatures are well below the sublimation temperature of carbon of about $4000 \mathrm{~K}$.

\section{Extreme Beam Conditions}

Figure 4 shows the measured foil current for a beam intensity of $5.2 \mu \mathrm{C} /$ pulse, injected over $625 \mu \mathrm{s}$. The storage time was increased from $0 \mu \mathrm{s}$ (no storage) to 400 , 600 and $800 \mu \mathrm{s}$. Consequently, the number of foil hits and the foil temperature were also increased. The measured foil current is very sensitive to the increase in foil temperature and is thus a valuable monitor of the strain put on the foil by extreme beam conditions.

We also deliberately damaged the foil by increasing the storage time to $1200 \mu \mathrm{s}$. There the TE current was about 25 times higher than the SE current. For these conditions we calculate a peak temperature in the hottest bin of about $4000 \mathrm{~K}$, i.e. the sublimation point of carbon in vacuum. Thermionic emission in this case lowers that temperature by about $100 \mathrm{~K}$. However, these numbers are preliminary as the calculation needs yet to be refined for this case.

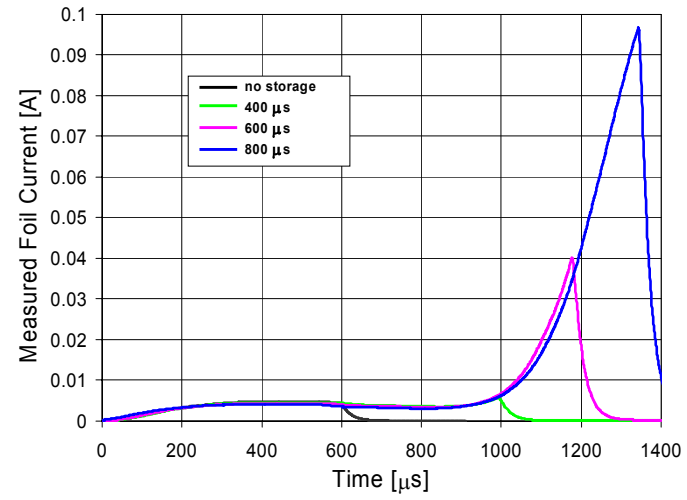

Figure 4: Measured foil current for different beam storage times.

\section{CONCLUSION}

Peak foil temperatures for PSR production beams (including future upgrades) are safely below the sublimation point of carbon.

At higher beam intensities or extended storage thermionic emission dominates the foil current, but does not seem to provide much additional cooling.

Thanks to the strong temperature dependence of TE monitoring the foil current can provide a very sensitive signal for safeguarding the foil from potentially severe beam conditions.

Improvements can be expected from a better understanding of the measured signals and refinements to the model and calculations.

\section{REFERENCES}

[1] M. Borden et al., "Carbon Stripper Foils Used in the Los Alamos PSR", Proceedings of PAC 1997.

[2] J. D. Galambos et al., "The ORBIT User's Manual", http://www.sns.gov//APGroup/Codes/orbit.htm. [3] National Institute of Standards and Technology, http://physics.nist.gov.

[4] J. E. Borovsky and D. M. Suszcynsky, "Experimental Investigation of the $\mathrm{z}^{2}$ Scaling Law of Fast Ion-produced Secondary Electron Emission", Physical Review A, Volume 43, Number 3, page 1416 (1991).

[5] A.T. Dinsdale, "SGTE Data for Pure Elements", CALPHAD 15, 317-425 (1991).

[6] High temperature data from "Thermophysical Properties of Matter - Nonmetallic Solids", TPRC Data Series.

[7] B. C. Lough et al., "Numerical Calculation of Thermionic Cooling Efficiency in a Double-Barrier Semiconductor Heterostructure", Physica E 11 (2001) 287-291. 